\title{
Experimental characterization of PCI impact on vibrating fuel rod under axial turbulent flow representative of JHR irradiation device ADELINE: Set-up conception and measurement method
}

\author{
Veronica D’Ambrosi ${ }^{\star}$, Stephane Breaud, Christophe Destouches, Guillaume Ricciardi, Frederic Lebon, \\ Emmanuelle Sarrouy, Jean-Marie Gatt, Jerome Julien and Daniel Parrat \\ * CEA, DEN, DER, Instrumentation Sensors and Dosimetry Laboratory, Cadarache, France, \\ veronica.d'ambrosi@cea.fr
}

\begin{abstract}
Mechanical loading originating due to PelletCladding Interaction (PCI) in PWR nuclear fuel rod constitutes a first order importance phenomenon when a ttempting at increasing the flexibility of $\mathbf{n}$ uclear $r$ eactors o peration $t$ of ollow grid demand. The need to improve the understanding of such complex multi-physics phenomena motivates the realization of particular irradiation sequences carried out in highly instrumented devices located in Material Testing Reactors. Among this kind, ADELINE irradiation device is being developed and will be installed in the future Jules Horowitz Reactor. In the perspective of designing the adapted measurement methodology to detect and characterize PCI phenomenology during irradiation, we present in this paper the experimental bench and its associated measurement program, designed to investigate eventual effects of PCI on the non-linear, flow i nduced $v$ ibrations of $\mathrm{n}$ uclear $\mathrm{f}$ uel $\mathrm{r}$ od. A nalytical and numerical models cannot predictively describe the system due to the complexity of phenomena thus the IMPIGRITIA experimental set-up has been developed to reproduce the mechanical interaction between the pellet and the clad at low pressure, room temperature and out of neutron flux. $T$ he $d$ esigned test bench presents a clamped free single short rod, centred in the test section by mean of four centring elements. Different rod configurations are implemented and localized closure of the gap is remotely realized by means of a dilatation system. Laser Doppler Vibrometry is used to measure the transversal displacement of the sample rod in three different conditions: in air, in stagnant water and under turbulent axial flow $r$ ate. $T$ he experimental program and expected results are presented and discussed.
\end{abstract}

Keywords-Nuclear, Instrumentation, Measurement, Bench, fuel rod, PCI, dynamics, vibrations, LDV

\section{INTRODUCTION: BACKGROUND AND OBJECTIVES}

$\mathbf{T}$ HROUGH the past years, Pellet-Cladding Interaction (PCI) has constituted an issue in the definition of technical specifications for the operation of nuclear power plants and for the identification of associated safety margins. Consequently, research has attempted to improve the knowledge

Manuscript received 2019; revised 2019.

V. D'Ambrosi, C. Destouches and S. Breaud were with the CEA, DEN, DER, Instrumentation Sensors and Dosimetry Laboratory, Cadarache, F-13108 St Paul Lez Durance, France. (email veronica.D'AMBROSI@cea.fr)

G. Ricciardi was with CEA Cadarache - DEN/DTN/STCP/LTHC, 13108 Saint Paul ls Durance, France

F. Lebon and E. Sarrouy were with Aix Marseille Univ, CNRS, Centrale Marseille, LMA, Marseille, France

J.M. Gatt, J. Julien and D. Parrat were with DEN/DEC, French Alternative Energies and Atomic Energy Commission (CEA) F- 13108 St-Paul-lezDurance - France of this complex phenomenon by numerical simulations, developing multi-physics models, and by experiments in Material Testing Reactors (MTR) [1]. In the latter, environmental constraints have strongly limited the possibility to obtain real time measurements and thus to allow characterisation of the kinetics of the phenomenology. At present days, a need for highly instrumented experiences in MTRs and for on-line measurements to gain real time data during irradiation has been expressed.

In the next future JHR, ADELINE is a specific irradiation device where PWR temperature and pressure conditions are reproduced and which will be partially dedicated at studying this phenomenology.

In this frame we have identified relevant and measurable parameters associated to PCI during normal irradiation and incidental conditions. We also proposed a model to characterize fuel material behaviour undergoing initiating central melting in the attempt at evaluating its macroscopic impact on PCI [2]. We are working to propose innovative and efficient measurement methods for detecting and characterizing this complex multi-physics phenomenology. Among the identified relevant parameters, vibration distinguished as a possible application case.

Flow Induced Vibrations (FIV) of nuclear fuel rods in axial turbulent flow rate have been concerned by a large variety of scientific studies. According to Païdoussis [3] [4] and Axisa [5] flow induced vibrations come from two mechanisms: the drag force in the turbulent boundary layer near the structure (nearfield component) and from the wall pressure fluctuations and far field noise. They generally induce transversal vibrations of small amplitudes, typically the vibration amplitude $\mathrm{y} / \mathrm{D}_{\mathrm{c}}$ does not exceed $10^{-3}$, y being the r.m.s. amplitude of vibration and $\mathrm{D}_{\mathrm{c}}$ the cylinder diameter. In the nuclear field, works are mainly motivated by fretting concern, phenomenon appearing at the grid-rod interface.

Few researches have instead been carried out on the effects of non linear mechanisms arising from the presence of pellets within the fuel rod. It is generally argued that modeling those effects is rather difficult [6]. Benhamadouche in [7] proposed to evaluate an equivalent mass density for the rod which accounts for the presence of pellets. This density is then used in the evaluation of the dynamic behaviour of the fuel rod taken as a linear Euler Bernoulli beam. Park et al. in [8] 
make the point on the fact that since a gap exists between pellets and gap, the contribution of the pellet mass to the density for dynamic evaluation cannot be clearly determined. They propose to compare the natural frequencies of empty and fulfilled rods to estimate an appropriate density.

The fuel rod vibration is then evaluated, still assuming a linear behaviour.

The non linear vibration of fuel rod has been recently studied by Ferrari et al. [6]: his study concerned a $900 \mathrm{~mm}$ zircaloy clad, clamped at both extremities, eventually fulfilled with cylindrical tungsten carbide pellets modelling uranium fuel pellets in terms of dimensions and density.

By comparing the dynamics of empty rod, fulfilled rod freely moving (no spring at one bottom of the fuel column) and fulfilled rod axially constrained in air and in quiescent water, they observed that pellets decrease natural frequencies of the rod and that axial constriction increases natural frequencies and reduced damping with respect to freely moving configuration, both in air and in water. Moreover, they stated that non linear mechanisms were experimentally observed during tests. No studies have been retrieved discussing the effect on the dynamics of the fuel rod of local pellet-clad contact due to gap closure. The impossibility to predictively model the non-linear system justified the design of the experimental set up IMPIGRITIA $^{1}$ to state the feasibility of measuring such impact.

This paper is structured as follows: we briefly proceed in section II with the numerical approach of the system, highlighting the empirical parameters to be measured to then present in section III the measurement principles.

Section IV is devoted to the design criteria of the experimental set-up, the definition of rod configurations and associated instrumentation. Section V then briefly describes the experimental program and expected results. Section VI proceeds to conclusions and future work.

\section{THE NUMERICAL APPROACH}

\section{A. Definition of the model}

Figure 1 shows a schematic view of the proposed model. The sample holder and the empty clad are modelled as a cantilevered Euler-Bernoulli beam by a 2D finite element model. Each node of the clad can move along the radial $\left(u_{x}\right)$ and the axial direction $\left(u_{y}\right)$ and can rotate $\left(\theta_{z}\right)$. Pellets are considered as discrete masses $m_{p}$ interacting along the radial direction with corresponding clad nodes through the non linear mechanism of dissipative shock and with each other through Coulomb friction. The equation of motion to be solved is [9]:

$$
[M]\{\ddot{x}\}+[C]\{\dot{x}\}+[K]\{x\}+\{f n l\}=\left\{F_{e}\right\}
$$

Where $[M]^{N, N}$ is the total mass matrix, $[C]^{N, N}$ the total damping matrix, $[K]^{N, N}$ the total stiffness one. They are obtained as follows:

$$
[M]=\left[\begin{array}{cc}
{\left[M_{c}\right]} & 0 \\
0 & {\left[M_{p}\right]}
\end{array}\right] \quad[C]=\left[\begin{array}{cc}
{\left[C_{c}\right]} & 0 \\
0 & {\left[C_{p}\right]}
\end{array}\right]
$$

\footnotetext{
${ }^{1}$ Acronym form 'Interaction Mécanique PastIlle Gaine :RéalIsation du Test vIbrAtoire", from latin impigritia, impigritiae; means "zeal, dedication"
}

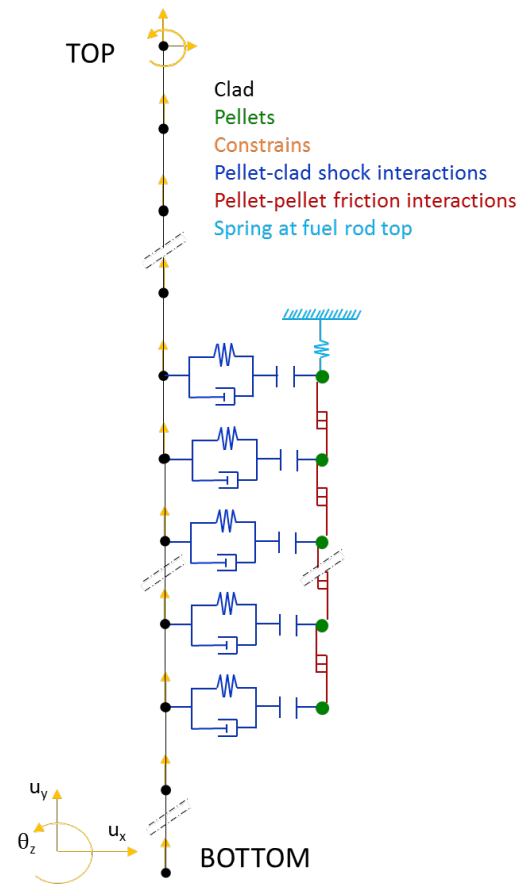

Fig. 1. Numerical model of IMPIGRITIA: schematic view of the configuration

$$
[K]=\left[\begin{array}{cc}
{\left[K_{c}\right]} & 0 \\
0 & {\left[K_{p}\right]}
\end{array}\right]
$$

where $\left[M_{c}\right]^{n, n}$ is the tridiagonal mass matrix for the clad and $\left[M_{p}\right]^{m, m}$ is the diagonal mass matrix for the pellets, with $N=m+n$. In the same way the total stiffness matrix and the total damping one depends on clad and pellets ones.

Rayleigh damping is used to model the damping matrix [C]:

$$
[C]=\alpha[M]+\beta[K]
$$

Assuming proportional damping, the dissipation in the structure can be described by two parameters $\alpha$ and $\beta$. Those parameters, which are not a priori known, are experimentally determined measuring the first two eigen-frequencies of the system in air. Indeed it can be shown that [10]:

$$
\zeta=\frac{1}{2}\left(\frac{\alpha}{\omega_{i}}+\beta \omega_{i}\right)
$$

where $\zeta$ is the modal damping, which can be recovered by logarithmic decrement from free damped vibrations of the system and $\omega_{i}$ are the eigen-frequencies.

The non-linear forces acting on the system are expressed by the $\{f n l\}$ term in equation (1). Those forces are the dry friction between discretized pellet nodes and the shock interaction between pellet nodes and clad ones. Gap size is a parameter in the formulation of pellet-clad shock interaction.

The presence of external water plays a role in the external solicitation $\left\{F_{e}\right\}$ but it also induces an additional viscous damping to the structure: according to De Pauw in [11] the effects of flowing fluid on the spectral response of the structure 
is described by an added mass $m_{a}$, corresponding to the mass of displaced fluid, and a dynamic amplification factor $C_{m}$ such that:

$$
f=\frac{1}{2 \pi} \sqrt{\frac{K}{M+C_{m} m_{a}}}
$$

where $\mathrm{K}$ and $\mathrm{M}$ are the structural stiffness and mass of the system. The dynamics amplification factor is equal to 1 for quiescent water thus the two parameters can be experimentally obtained by comparing the oscillation frequencies of the system in air, in quiescent water and in fluid flow.

$\left\{F_{e}\right\}$ corresponds to the excitation mechanisms acting on the system, notably the flow solicitation. We decided to apply a statistical approach and model $\left\{F_{e}\right\}$ by taking the envelop PSD defined by Axisa [5] and applying an arbitrary stochastic phasing.

To solve the equation, explicit Newmark scheme has been implemented. As reported in [9] direct time integration is necessary when the problem is non linear in order to avoid strong dependencies of results on integration time step.

\section{B. Investigation of parameters: an empirical model}

The displacement $\{x\}$ of the structure, obtained when solving motion equation (1) by mean of explicit Newmark integration scheme, can be expressed as a function of the set of parameters $\mathbb{P}$, resulting from the previous discussion:

$$
\begin{aligned}
& \{x\}=f\{\mathbb{P}\}= \\
& f\left\{\mathbf{M}_{\mathbf{p}}, \mathbf{M}_{\mathbf{c}}, m_{a}, C_{m}, \mathbf{K}_{\mathbf{p}}, \mathbf{K}_{\mathbf{c}}, \alpha, \beta, K_{c}, C_{c}, \mu, j, c^{2}\right\}
\end{aligned}
$$

where $\mathbf{M}_{\mathbf{i}}, \mathbf{K}_{\mathbf{i}}, \alpha$ and $\beta$ have already been defined, $m_{a}$ and $C_{m}$ are the added mass and the dynamic amplification factor due to the fluid presence and fluid flow. $K_{c}$ and $C_{c}$ are the parameters characterising the pellet-clad shock interaction, modelled through a contact stiffness $K_{c}$ and a viscous dissipation $C_{c}$ [12]; $\mu$ is the friction coefficient to model the pellet-pellet interaction. $j$ is the width of the gap and $c$ is the fluid velocity.

While some of those parameters are completely defined by the geometry of the structure (i.e. the mass matrix of pellets $\mathbf{M}_{\mathbf{p}}$ and sample holder $\mathbf{M}_{\mathbf{c}}$, the stiffness matrix of the sample holder), the others have to be experimentally determined. Identification of model parameters adapted to fit at best experimental data allows calibration of the model. They are resumed in table I.

\section{THE MEASUREMENT PRINCIPLE}

The presence of pellets inside the clad induces the appearance of non linear mechanical dissipations [6]. Internal attachment between pellets and clad as a consequence of gap closure limits those effects and impacts mode and frequencies of the system. We expect local and progressive mechanical contact between pellets and clad to reduce structural damping as a consequence of reduced dissipative mechanisms and to decrease its eigen-frequencies. Those variations can be retrieved by measuring the displacement of the system $x(t)$. Experimental identification of the frequencies and of modal properties, as the modal damping, can be carried out both in
TABLE I

MODEL PARAMETERS

\begin{tabular}{ll}
\hline Parameter & Description \\
\hline $\mathrm{K}_{p}$ & Pellet Stiffness \\
$\zeta(\alpha, \beta)$ & Modal damping \\
$m_{a}$ & Added mass \\
$C_{m}$ & Dynamic amplification factor \\
$K_{c}$ & Contact Stiffness \\
$C_{c}$ & Contact viscous dissipation \\
$\mu$ & Pellet-Pellet Friction Coefficient \\
$j$ & Pellet-clad gap \\
$c$ & Fluid velocity \\
\hline
\end{tabular}

the time domain or in the frequency one through direct or inverse Fourier transform. Indeed:

$$
x(t) \leftrightarrow \quad X(\omega)
$$

where $X(\omega)$ is the Fourier transform of the time signal of the displacement $x(t)$ of the point characterized by the highest transversal displacement. $X(\omega)$ and $x(t)$ are linked by the relations:

$$
\begin{array}{r}
X(\omega)=\mathcal{P}[x(t)]=\int_{-\infty}^{\infty} x(t) e^{-i \omega t} d t \\
x(t)=\mathcal{P}^{-1}[X(\omega)]=\frac{1}{2 \pi} \int_{-\infty}^{\infty} X(\omega) e^{i \omega t} d \omega
\end{array}
$$

In both time and frequency domains, system identification shall be carried out on the basis of the model presented in this section, adapting its parameters to fit at best the experimental data. Several methods are available in literature [9] and [11]. The impact of pellet-clad interaction on the transversal vibration $x(t)$ can be experimentally obtained by comparing specifically designed rod configurations under the same excitation conditions. Calibration of parameters previously discussed allows validation of the model capability to simulate the dynamic response of the structure.

\section{DESIGN CRITERIA}

\section{A. Objectives}

We have manufactured the experimental test bench to state the capability to measure whether gap closure impacts fuel rod dynamics. We want to identify if a minimum number of pellets has to interact with the clad to have a measurable effects and to define if FIV are sufficient to have a passive measurement and to study the influence of this axial flow excitation on the feasibility of this measure. Application domain is the ADELINE device.

\section{B. The representativeness issue}

ADELINE [13] is designed to be a $4 \mathrm{~m}$ high irradiation device and constitutes the evolution of ISABELLE 1 system as it was in OSIRIS reactor [14]. As its predecessor, it presents a single rod test section where temperature and pressure conditions characteristics of a PWR are reproduced (155 bar and 
$275^{\circ} \mathrm{C}$ inlet water temperature). The tested rod has geometrical characteristics of FABRICE type fuel-rod [14]: a short element (about $60 \mathrm{~cm}$ in length) obtained cutting a previously irradiated $4 \mathrm{~m}$ fuel rod and adapted to MTR geometry. The rod is cooled by an up-streaming turbulent flow rate. It is maintained in the section by a sample holder and centred by four centring elements.

In order to be representative of ADELINE configuration, we accounted for the real environmental conditions, for the geometrical design, material characteristics and hydraulic solicitation.

1) The environment: Thermal and mechanical properties are obviously impacted by the environmental conditions but, through the years, several correlations have been developed to account for these phenomena. Those correlations will be used during post treatment of results to establish the attended behaviour in PWR conditions [15].

The pressurization of the circuit has no impact on the vibration of the component, thus leading to design a law pressure system.

2) The geometry: Mechanical similarity and scale reduction cannot simply be applied and a 1:1 scale system implied non negligible conception, exploitation and cost issues. Still, geometry and fixation conditions strictly impact the dynamics of a structure thus the clamped free conditions of ADELINE sample is maintained in the experimental set-up. Moreover, ADELINE sample holder is made of an upper part and a lower part, the sample rod a centring elements being located in this last one. The two parts are coupled by a mechanical system. The specific configuration and material properties of the real system lead us to decide to limit our study to the lower sample holder part. It results an experimental system of about $1.5 \mathrm{~m}$. This choice is expected to strengthen the impact of a local pellet-clad interaction on the global dynamics of the structure.

3) The hydraulic similarity for FIV: Fuel rod to be installed in the ADELINE device will be submitted to a turbulent flow rate of approximately $1 \mathrm{~m} / \mathrm{s}$ at about $275^{\circ} \mathrm{C}$ and 155 bar along the axial direction, from the bottom to the top. Such kind of turbulent flow rate induces the appearance of FIV of the sample rod. Hydraulics is dimensioned in order to reproduce the external solicitation in terms of the axial turbulent flow and pressure fluctuation in the flow. We identified a range of representativeness first by imposing the conservation of wall pressure fluctuations and secondly applying the Burgreen principle. The conservation of wall pressure fluctuation $p_{r . m . s}$ was imposed according to the definition proposed by Axisa in [5] on the basis of the envelop PSD derived from Clinch data [16] and reported in equation (9). The simulation criterion established by Burgreen [17] instead has been recently used by De Paw et al. in [11] to study flow induced vibrations in a prototype representative of the MHYRRA system and it is reported in equation (10).

$$
p_{\text {r.m.s }}=0.003\left(\rho c^{2}\right)
$$

where $p_{\text {r.m.s }}$ is the root mean square of pressure fluctuations, $\rho$ is fluid density and $c$ is average flow velocity.

$$
c_{\text {exp }}=c \frac{\rho^{0.5} \mu^{-0.33} E^{-0.33} M^{0.165}}{\rho_{\text {exp }}^{0.5} \mu_{\text {exp }}^{-0.33} E_{\text {exp }}^{-0.33} M_{\text {exp }}^{0.165}}
$$

Subscript 'exp' stands for prototype, $\mu$ the fluid dynamic viscosity, $E$ elastic modulus and $M$ the mass of the sample rod at operating temperature.

Applying the described criteria in our test conditions we found a range of representative flow rate varying between 0.9 to 2.6 the fluid velocity at the sample rod in ADELINE.

$$
\frac{c_{e x p}}{c} \in[0.9: 2.6]
$$

\section{THE EXPERIMENTAL SET-UP AND PROGRAM}

For the experience several representative hollow zircaloy clad were supplied. As previously mentioned, the geometrical configuration of sample rods was defined on the basis of FABRICE type fuel rods used in ADELINE: the zirconium alloy clad has an external diameter of $9.5 \mathrm{~mm}$, its material properties are reported in table II. We defined a length coherent with FABRICE characteristics thus $550 \mathrm{~mm}$ long. Each side is welded to a plug which is screwed on a prolongation tube in stainless steel.

The prolongation tube constitutes the sample holder and it is fixed at the top of the experimental section. The other side is instead free to move. A simplified scheme of the sample holder is presented in figure 2 .

Due to environmental and realisation issues, the $\mathrm{UO}_{2}$ constituting the real fuel material was replaced by cylindrical Molybdenum pellets, which is one of the most representative materials according to internal researches carried out at CEA. A comparison of room temperature material properties of $\mathrm{UO}_{2}$ and Mo are resumed in table II. Pellets diameter was defined according to standard radial dimension of $\mathrm{UO}_{2}$ fuel pellets, the height instead was deduced by mass conservation of $\mathrm{UO}_{2}$ fuel pellets.

In order to fulfil the fabrication gap existing between pellets and clad and thus remotely control PCI, a dilatation system capable of local expansion is used. The tool, showed in figure 3 , is used in the medical domain for stent implantation: it is made of a balloon which can be externally inflated and a connection tube (the shaft) allowing positioning and inflation of the system.

The component is introduced from the top of the sample holder, through centrally drilled pellets and the balloon is placed in the region where gap closure wants to be induced. There, pellets are replaced by two shells which are displaced by the dilatation of the system, leading to pellet-clad contact.

TABLE II

MATERIAL PROPERTIES OF INTEREST

\begin{tabular}{ccc}
\hline Material & $\begin{array}{c}\text { Density } \\
{\left[\mathrm{kg} / \mathrm{m}^{3}\right]}\end{array}$ & $\begin{array}{c}\text { Young's Modulus } \\
{[\mathrm{GPa}]}\end{array}$ \\
\hline Zircaloy & 6570 & 95 \\
$\mathrm{UO}_{2}$ & 10963 & 194.3 \\
Mo & 10200 & 320 \\
\hline
\end{tabular}




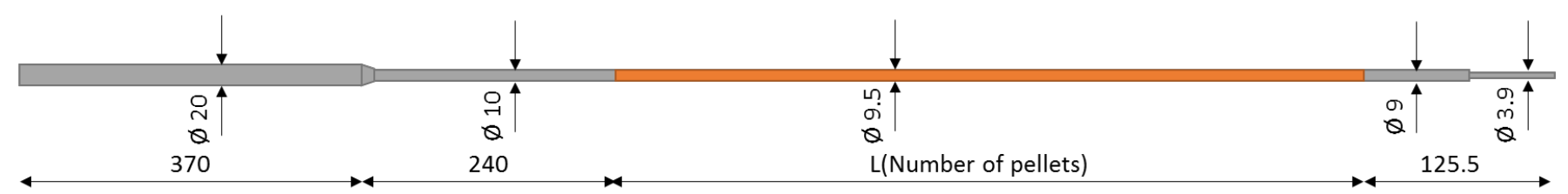

Fig. 2. Sample holder scheme

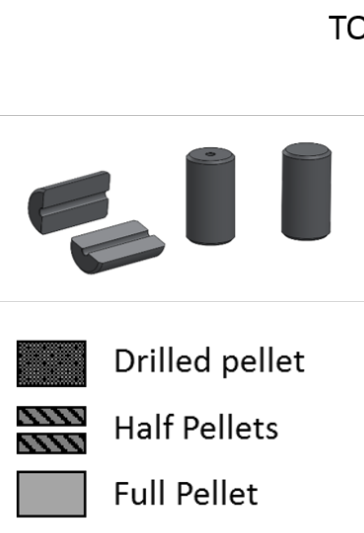

TOP

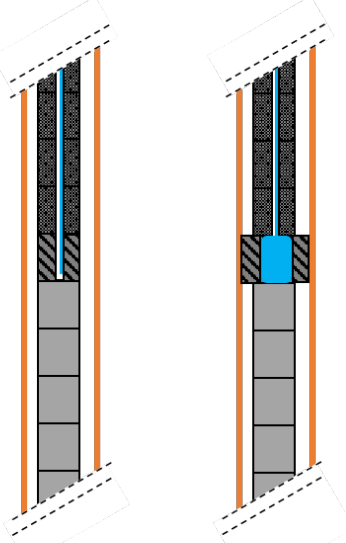

BOTTOM

Fig. 3. Schematic view of the functioning of expansion system to induce pellet-clad interaction: inflation of the balloon through the shaft leads to fakepellets displacement inducing pellet-clad contact in a localized region of the sample rod.

TABLE III

ROD CONFIGURATIONS

\begin{tabular}{ccc}
\hline Rod name & $N^{\circ}$ of displaced pellets & Axial extension of gap closure \\
\hline C2 & 1 & $15 \mathrm{~mm}$ \\
C3 & 3 & $45 \mathrm{~mm}$ \\
C4 & 5 & $75 \mathrm{~mm}$ \\
C5 & 9 & $135 \mathrm{~mm}$ \\
\hline
\end{tabular}

We decided to test different rod configurations taking advantage of different length of balloons in order to identify the minimum number of contacting pellets leading to a measurable effects. Rod configurations are resumed in table V. To calibrate the model and characterize system dynamics response, three other rod configurations were manufactured: an empty rod C0 to characterize modal parameters governing the linear dynamic response of the sample rod; $\mathbf{C 1}$ which corresponds to the fuel rod being fulfilled with Mo pellets, allowing to highlight possible bias due to the presence of dilatation system; and C6, where Mo pellets and clad are designed to stick together. This last configuration allows to measure to effect on sample rod dynamics due to complete closure of the gap.

As previously mentioned, the sample rod is maintained by a sample holder, centered in the experimental section by mean of four elements. In order to collect vibration data of the sample rod from Laser Doppler Vibrometer (LDV) system, the experimental section is completely designed in optical quality plexiglass. This type of non-contact measuring system is capable of collecting the vibration of structures even in the presence of surrounding water.

Specifically designed holders allow axial translation of sensible heads. This allows for reconstruction of mode shapes of the rod and experimental modal analysis. The measurement system is showed in figure 4.

To produce and control flow conditions in the experimental section we have designed a dedicated independent water loop. as shown in figures 5 and 6 . All conditioning components are located on a separate frame to limit noise effect on LDV measures. To monitor conditions in the circuit we have installed a flow meter at the experimental section inlet, three thermocouples located at different positions to verify temperature evolution in the loop and three pressure gauges to check pressure drops. A summary of instrumentation characteristics and accuracy is reported in table IV.

Measures during the experience are carried out in three different conditions: in air, in quiescent water and under axial, turbulent flow rate. When the system is in air and in stagnant water, the excitation technique is constituted by a magnetic solicitation system attracting the final section of the sample holder, especially made of ferromagnetic material. Logarithmic decrement applied on the collected signal of the underdamped system is used to obtain its damping ratio. Test of the different rod configurations in air and in stagnant water leads

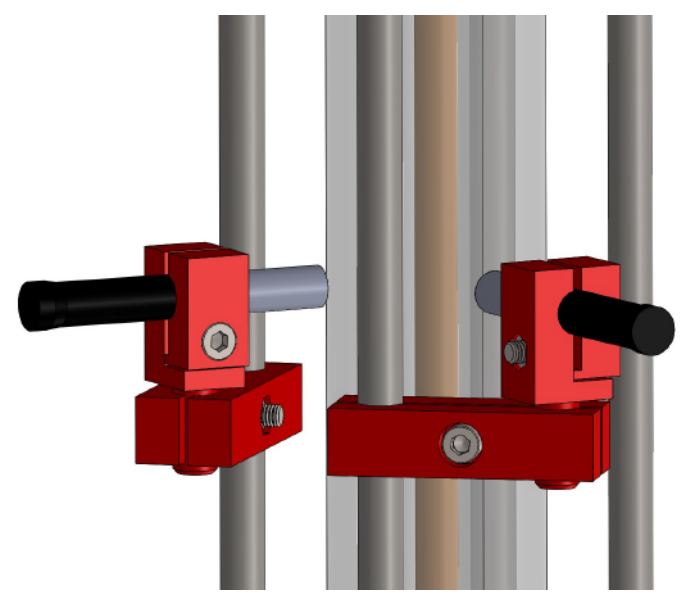

Fig. 4. View of measurement system by LDV: the clad, highlighted in orange, is visible through the plexiglass experimental section; two metallic rods helps supporting the two red structures holding laser heads. The two laser heads located at $90^{\circ}$ lead to verify axisymmetric vibrations of the rod 
TABLE IV

INSTRUMENTATION

\begin{tabular}{cccc}
\hline Parameter & $\mathrm{f}[\mathrm{Hz}]$ & Instrument & Accuracy \\
\hline Vibration & $1-10^{4}$ & Laser Doppler Interfermeter & $10^{-7} \mathrm{~m} / \mathrm{s}$ \\
Temperature & 1 & K type thermocouples & $+/-1.5 \mathrm{~K}$ \\
Pressure & 1 & pressure gauges & $+/-0.3$ bars \\
Flow rate & imposed & Vortex effect flow meter & $+/-21 / \mathrm{min}$ \\
\hline
\end{tabular}

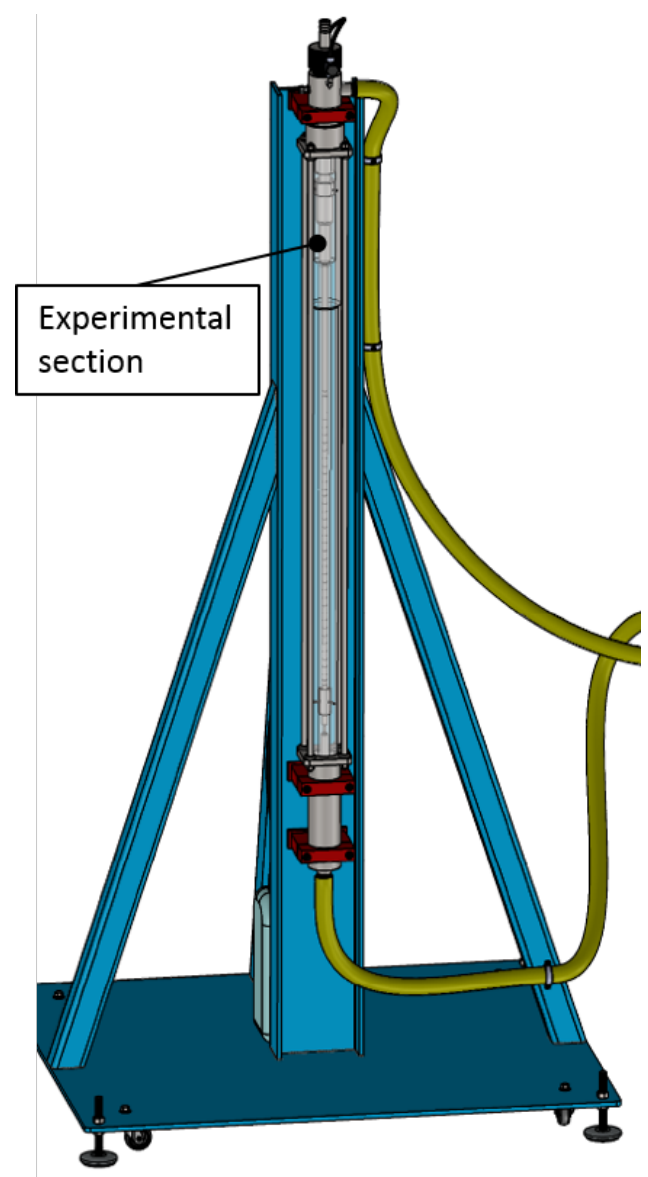

Fig. 5. View of designed experimental test section located on the supporting frame

to the calibration of the unknown parameters defined in section II-B and to verify the possibility of measuring PCI under active solicitation. The test under flow rate excitation then leads first to characterize the amplification factor due to fluid presence and then feasibility of measuring the phenomenon according to a passive approach.

\section{CONCLUSIONS AND FURTHER WORK}

The IMPIGRITIA test bench was designed to investigate PCI and innovative measurement methods. The experiment is conceived to verify the capability of measuring the modification of modal properties and dynamic response as a function of the partial closure of internal pellet-clad gap under turbulent flow excitation representative of PWR environment.

Open literature supports the fact that the non linear mechanisms acting on the dynamics of the fuel rod structure

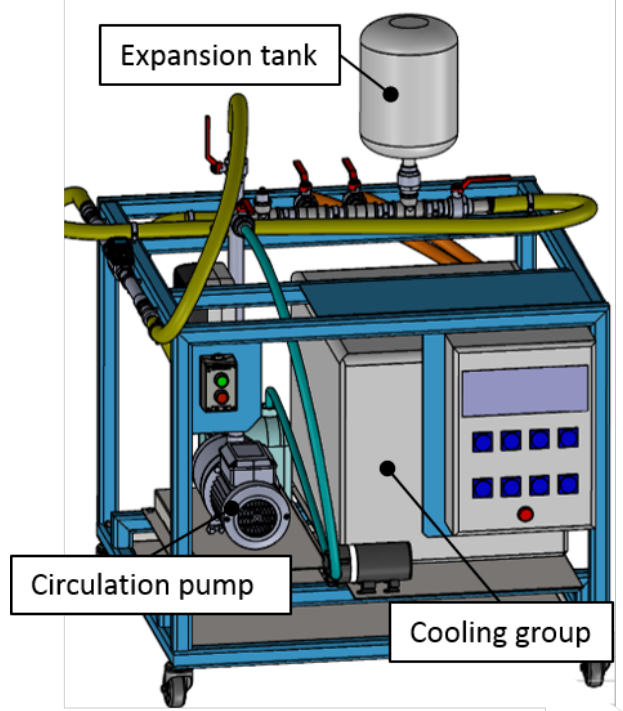

Fig. 6. View of designed water loop conditioning the experimental section

induce measurable variations: the closure of the gap causes a global increase in the isotropy of the dynamic response and a reduction in damping and frequencies of the structure.

The system has been designed though material, mechanical and hydraulic similarity, to reproduce mechanical PCI at room temperature, at low pressure and being representative of the geometry of ADELINE device. Some modifications in the geometry have been carried out which enhance the possibility of measuring the investigated effects.

Due to environmental issues, $\mathrm{UO}_{2}$ cannot be used in such experiments: the identified representative material is Molybdenum.

According to literature, transversal vibrations in axial turbulent fluid flow are due to pressure fluctuations in the fluid pressure field: hydraulic similarity has been applied and a range of flow velocities varying between [0.9:2.6] ADELINE's one has been identified, using the conservation of pressure fluctuation and the Burgreen principle.

An expansion system has been chosen to locally induce gap closure.

To understand and simulate the physics of the dynamic response of the structure, a model has been developed. It cannot be predictive for the phenomenon under analysis as a large set of parameters has to be first experimentally calibrated. The experimental program has thus been defined to cope with calibration issues, testing in different experimental conditions (air, stagnant water and turbulent axial flow) several rod configurations, notably an empty rod, a fulfilled rod without dilatation system, four rods having PCI axial extension progressively increasing and a full rod with pellets stuck to the clad.

We will characterize vibration mode in terms of damping ratio and eigen-frequencies by measuring the transversal displacement of the system under active controlled solicitation and under passive flow induced vibration. We expect that a 
correlation could exists, coupling the axial extension of gap closure with the damping modification of the structure.

Whether a measurable modification is clearly identified, we shall proceed to a study to evaluate an adapted instrumentation methodology to cope with ADELINE configuration and nuclear environment, where it would lead to a better understanding of the PCI mechanism under irradiation.

\section{REFERENCES}

[1] B. Michel, J. Sercombe, C. Nonon, F. Michel, and V. Marelle, "Simulation of Pellet-Cladding Interaction with the PLEIADES Fuel Performance Software Environment," Nuclear Technology, vol. 182, pp. 124-137, 2013.

[2] V. D'Ambrosi, J. Gatt, F. Lebon, J. Julien, D. Parrat, and C. Destouches, in Proceedings of MECAMAT 2018, 2018.

[3] M. P. Païdoussis, Fluid-Structure Interactions, Slender Structures and Axial Flow. ELSEVIER, 2004, vol. 2.

[4] M. P. Paidoussis, "Fluidelastic vibration of cylinder arrays in axial and cross flow: State of the art," Journal of Sound and Vibration, vol. 76, no. 3, pp. 329-360, 1981. [Online]. Available: http://www.sciencedirect.com/science/article/pii/0022460X81905162

[5] F. Axisa, Modélisation des systèmes mécaniques : Vibrations sous écoulements. Hermes, 2001, vol. 4.

[6] G. Ferrari, P. Balasubramanian, and al., "Non-linear vibrations of nuclear fuel rods," Nuclear Engineering and Design.

[7] S. Benhamadouche and al., "CFD estimation of the flow induced vibrations of fuel rod downstream a mixing grid," proceeding of the ASME 2009 Pressure vessels and Piping division conference.

[8] N. Park, H. Rhee, J.-K. Park, S.-Y. Jeon, and H.-K. Kim, "Indirect estimation method of the turbulence induced fluid force spectrum acting on a fuel rod," vol. 239, no. 7, pp. 1237-1245. [Online]. Available: http://www.sciencedirect.com/science/article/pii/S0029549309001320

[9] M. Gradin and D. J. Rixen, Mechanical Vibrations, Theory and Application to Structural Dynamics., third edition ed. Wiley.

[10] A. Alarcon, "Code aster: Modeling of damping in dynamics linear."

[11] B. De Pauw, W. Weijtjens, S. Vanlanduit, K. Van Tichelen, and F. Berghmans, "Operational modal analysis of flow-induced vibration of nuclear fuel rods in a turbulent axial flow," Nuclear Engineering and Design, vol. 284, pp. 19-26, Apr. 2015. [Online]. Available: http://www.sciencedirect.com/science/article/pii/S0029549314006694

[12] P. Wriggers, Computational Contact Mechanics, second edition ed. Springer Berlin Heidelberg New York.

[13] C. Gonnier, J. Estrade, G. Bignan, and B. Maugard, in Proceedings of IGORR 2017, 2017.

[14] A. Alberman, M. Roche, P. Couffin, S. Bendotti, D. Moulin, and J. Boutfroy, "Technique for power ramp tests in the isabelle 1 loop of osiris reactor," Nuclear Engineering and Design, vol. 168, no. 1, pp. 293-303.

[15] J. K. Fink, "Thermophysical properties of uranium dioxide," vol. 279, pp. $1-18$.

[16] J. M. Clinch, "Measurements of the wall pressure field at the surface of a smooth-walled pipe containing turbolent water flow," Journal of Sound and Vibration, vol. 9, pp. 398-419, 1969.

[17] V. Prakash, M. Thirumalai, M. Anandaraj, P. A. Kumar, D. Ramdasu, G. Pandey, G. Padmakumar, C. Anandbabu, and P. Kalyanasundaram, "Experimental qualification of subassembly design for prototype fast breeder reactor," vol. 241, no. 8, pp. 3325-3332. [Online]. Available: http://linkinghub.elsevier.com/retrieve/pii/S0029549311003487 